\title{
Impact of Anesthesia on Systemic and Cerebral Glucose Metabolism in Diabetes Patients Undergoing Neurosurgery "Updates of Diabetes and Neurosurgical Anesthesia"
}

Jiang Tao and Liu Youtan*

Department of Anesthesiology, The University of Hong Kong Shenzhen Hospital, Hong Kong

\begin{abstract}
Accumulating evidences demonstrate that patients with diabetes have higher perioperative risk of complications and longer hospital stays than patients without diabetes. Intensive insulin therapy (IIT) may improve outcomes in hyperglycemic neurosurgical patients. However, currently there is no adequate data/evidence regarding optimal insulin delivery protocols and targeted blood glucose concentration during IIT. IIT continues to be explored as a therapeutic method to reduce morbidity associated with derangements in glucose metabolism. Hypoglycemia is a common side effect of IIT with the potential to result in significant morbidity, especially in patients undergoing neurosurgical surgery. Surgical stimulus is a potent cause which may affect patients stress response and influences the metabolic and endocrine system, which can lead to electrolyte imbalance and decrease of blood insulin level and subsequently result in impairment of immune response and increase of blood glucose level. The stress response can be prevented by sufficient Depth of anesthesia. So the Anesthesiologists play a very important role in manipulating the blood glucose of the diabetes patients during the neurosurgical operations. Narcotics affect systemic and cerebral metabolism, and studies indicate that most of anesthetics have restraining effects on brain oxygen and glucose consumption. In order to better regulate the blood glucose, the anesthesiologists should master the actions of every anesthetics. IIT is a double-edged sword; more investigations should be focused on optimising blood glucose level for euglycemia maintenance in the intraoperative management of diabetes neurosurgical patients.
\end{abstract}

Keywords: Diabetes patients undergoing neurosurgery; Perioperative blood glucose stability; Impact of anesthesia on diabetes patients; Anesthesia managements

\section{Introduction}

The prevalence of diabetes mellitus is augmenting rapidly in the 21st century mainly due to obesity, aging and the lacking of exercise. The International Diabetes Federation (IDF) declared in 2008, that 246 million adults had diabetes mellitus (DM) worldwide and the prevalence was expected to reach 380 million by 2025 [1]. Patients with diabetes preparing for surgery are facing many difficulties. They may have vascular, renal, or neurological disease as a consequence of their underlying DM and are more prone to wound infections. Maintaining normal blood glucose concentrations has been shown to reduce perioperative morbidity and mortality, although the evidence for this derives mainly from studies in patients undergoing cardiac surgery [2]. But a recent retrospective study found that hyperglycemia was relevant to increased length of hospital stay, morbidity, and mortality after noncardiac surgery in diabetic and non-diabetic patients [3].

Hyperglycemia with simultaneous cerebral ischemia is associated with detrimental clinical sequelae in some neurosurgical patients. These include a longer intensive care unit stay, poor recovery of neurologic function, symptomatic cerebral vasospasm, and increased cerebral infarct size [4]. Intensive insulin therapy (IIT) may improve outcomes in hyperglycemic neurosurgical patients. The adoption of IIT has been limited by a lack of adequate data on optimal insulin delivery protocols and serum glucose concentration and by concerns about the risks of hypoglycemia [5]. Therapeutic interventions to lower glucose levels to a tight range $(80-120 \mathrm{mg} / \mathrm{dl})$ with intensive insulin therapy (IIT) have been studied in a variety of critical care settings with mixed results [4]. However, more recent data suggest that there exist possible harmful effects of IIT on brain tissue. To date, solid clinical evidence to justify IIT in neurocritically ill patients does not exist [6,7]. Caution is necessary when generalizing IIT clinical studies from critical ill patients to neurosurgical patients since brain glucose metabolism is often altered by neurological injury [4]. Clinical data regarding glucose control in the general surgical population and during neurocritical care have been reviewed $[8,9]$.

As the diabetic population is keeping growing worldwide, anesthesiologists may have more chances to encounter patients with DM in the management of perioperative period. Non-diabetic patients may become hyperglycemic state due to a combination of tissue insulin resistance and decreased insulin secretion in the perioperative period. In diabetic patients, surgery and trauma are associated with an increase in the secretion of catabolic hormones in the presence of relative insulin deficiency. Therefore, the aim of perioperative metabolic management should be targeted to avoid excessive hyperglycemia, hypoglycemia and loss of electrolyte such as potassium, magnesium and phosphate $[10,11]$.

Based on the fact that there will be more diabetes neurosurgical patients needing anesthesia, anaesthetists will be more involved in the perioperative care, as the number of these patients keep growing. Little is known about the relationship between anaesthesia and preoperative

*Corresponding author: Liu Youtan, Department of Anesthesiology, The University of Hong Kong Shenzhen Hospital, University of Hong Kong, Shenzhen, Guangdong 518000 P.R, Hong Kong, E-mail: liuyt@hku-szh.org

Received July 29, 2013; Accepted September 17, 2013; Published Septembe 24, 2013

Citation: Tao J, Youtan L (2013) Impact of Anesthesia on Systemic and Cerebral Glucose Metabolism in Diabetes Patients Undergoing Neurosurgery "Updates of Diabetes and Neurosurgical Anesthesia". J Diabetes Metab 4: 297. doi:10.4172/2155-6156.1000297

Copyright: @ 2013 Tao J, et al. This is an open-access article distributed under the terms of the Creative Commons Attribution License, which permits unrestricted use, distribution, and reproduction in any medium, provided the original author and source are credited. 
glycemic state in diabetes neurosurgical patients. Improved understanding of this relationship may identify patients at increased risk of complicated recovery and guide postoperative treatment strategies. This article will review the impact of anaesthesia on systemic and cerebral metabolism of glucose in diabetes neurosurgical patients.

\section{Fluctuations of Blood Glucose during the Perioperative Period in Neurosurgury of DM Patients}

Many factors affect the blood glucose of DM patients during the perioperative period in neurosurgery. Factors such as trauma, hemorrhage, ischemia, infection, surgery stress, anesthetic agents, critical illness and metabolic stress may lead to insulin resistance and B-cell dysfunction. These factors may cause the increase in blood glucose [12]. The stress of surgery may be the main factor. It activates a neuroendocrine response that may function against the action of insulin. Stress also evokes the development of insulin resistance, which can be caused by proinflammatory cytokines $[13,14]$ and other factors such as some drugs. Stress-induced hyperglycemia may induce endothelial cell dysfunction, defects in immune function, aggravated oxidative stress, prothrombotic changes, cardiovascular effects, and specific brain area injury or a direct hypothalamic damage/irritation of glucose regulatory centers [12,15-17]. Hyperglycemia has been shown to exasperate these deleterious effects.

Hypoglycemia is defined as a blood glucose value of $<70 \mathrm{mg} / \mathrm{dL}$ ( $<3.9 \mathrm{mmol} / \mathrm{L}$ ) [18]. Hypoglycemia is a common, multifactorial, and evitable episode. It can occur in any case, and the DM patients are the most sensitive. There is no definite data about its occurrence in neurosurgical DM patients, but it is generally known that Hypoglycemic events deteriorate prognosis [19-21]. The Hypoglycemia associated risk is greater during the perioperative period, because general anesthesia may mask the symptoms and postpone its recognition.

\section{Glucose Metabolism of the Brain}

A continuous supply of both oxygen and glucose is vital for normal metabolic function of the brain. It includes biochemical synthesis, synaptic transmission, and maintenance of transmembrane ionic gradients and cellular integrity. The brain contains manifold cell types (e.g., neurons, glial cells such as astrocytes, oligodendrocytes). Each of these cell types appears to have different metabolic features and, probably different vulnerabilities [4].

Glucose delivered to the brain is taken up into cells and undergoes glycolytic breakdown to adenosine tri-phosphate (ATP) and pyruvate under aerobic conditions (i.e., oxygen available and mitochondrial function is normal). Pyruvate is transformed to acetyl-CoA via the tricarboxylic acid (TCA) cycle to generate ATP and reducing equivalents. When oxidative phosphorylation is impaired (lack of oxygen, mitochondrial dysfunction), pyruvate is converted to lactate instead. Most of these biosynthetic reactions are under tight feedback control. Delicate equilibriums are modulated by many factors including acid-base status, redox status, and substrate availability/concentration [4].

Normal brain glucose metabolism is modified under conditions of hyperglycemia, hypoglycemia, and pharmacologic therapy. Animal and human researches have demonstrated that there is a significantly altered glucose metabolism in the setting of traumatic brain injury, subarachnoid hemorrhage, and cerebral ischemia [22]. The metabolic disturbances associated with neuro-pathology are heterogeneous and continue to change with the evolution of the underlying pathologic process [4].

\section{Intensive Insulin Therapy and Outcome in Neurosurgical Patients}

Since the development of intensive insulin therapy for the DM patients, tight glycemic control (TGC) has become increasingly complicated to apply and achieve. Short term intensive insulin therapy has been reported to induce long term euglycemia remission in DM patients, but the factors that are responsible for long-term remission or hyperglycemia relapse are unknown. There has been a great deal of interests for intensive glycemic control after landmark researches by Van Den Berghe and colleagues [23,24]. The initial prospective randomized single-center study of surgical intensive care unit patients included a small number of brain-injured patients. The overall results indicated convincingly that intensive glycemic control was beneficial, and subsequent post-hoc analysis indicated that intensive glycemic control resulted in less neurologic complications [25].

The up-to-date medical intensive care unit study of intensive glycemic control was less convincing [26]. In the latter study, mortality was not reduced in the overall intention-to-treat group, and a very small positive effect of intensive glycemic control on mortality was seen in patients treated for $>3$ days. At the same time, there remain some concerns that intensive glycemic control $(4.4-6.1 \mathrm{mmol} / \mathrm{L})$ is not proper for brain-injured patients, and may induce secondary injury [27]. Using cerebral microdialysis in brain-injured patients, Vespa and colleagues found that the infusion of insulin to achieve intensive glycemic control resulted in profound reductions of brain glucose and elevations of biomarkers of cellular distress (such as glutamate and lactate/pyruvate ratio). This finding has been replicated in patients with subarachnoid hemorrhage [28], in which patients with intensive glycemic control have elevated microdialysis glycerol and elevated lactate/pyruvate. In addition, there is evidence that moderate hyperglycemia (12-15 $\mathrm{mmol} / \mathrm{L})$ is not associated with adverse events after brain injury $[25,29]$.

A small single-center randomized experiment comparing intensive glycemic control with moderate hyperglycemia in subarachnoid hemorrhage patients recently failed to demonstrate beneficial effect on mortality or vasospasm ischemic insults [30]. Therefore, there is great uncertainty about how best to control systemic glycemia after brain injury [25]. Given the cumulative evidence that intensive glycemic control is associated with metabolic distress [27], with increased hypoglycemia, with worsened intracranial pressure, and with worsened mortality [31] - the ideal range for glycemic control is not clear.

\section{Impact of Anaesthesia on Diabetes Neurosurgical Patients}

DM is a risk factor for suboptimal perioperative outcomes in patients undergoing neurosurgery $[32,33]$. Several studies have identified an association between diabetes and infectious complications after major spine surgery [34]. Patients with DM also have an increased frequency of complications, both major (e.g., wound infection, peripheral nerve root lesion, cardiac arrhythmia, acute renal failure, cerebrovascular accident) and minor (e.g., urinary tract infection, paralytic ileus, electrolyte deficiencies) [12].

Anesthesiologists have more chances to confront diabetic patients during the perioperative period. In diabetic patients, surgery and trauma are associated with an increase in the secretion of catabolic hormones due to the relative insulin deficiency. Therefore, the goal of perioperative metabolic management is to avoid excessive hyperglycemia, hypoglycemia [33]. 
Some researches show that long-term intensive glycemic control in type 2 diabetic patient significantly reduced microvascular complications [11,34]. A study [35] showed that perioperative hyperglycemia is associated with increased hospital length of stay, hospital complications, and mortality after noncardiac general surgery. Especially, in this study, the rate of postoperative complications such as pneumonia, wound infection, urinary tract infection, acute myocardial infarction, and acute renal failure, was higher in diabetic patients than nondiabetics during the hospital stay [36] which argued for maximal benefit in the range of 144 to $180 \mathrm{mg} / \mathrm{dl}$. Therefore, even if these studies are postulated that insulin therapy is not harmful, and may even be beneficial, it remains unclear what targets of glycemic control are appropriate. It is definite that tighter control necessitates more insulinmediatedpotential harmful effects, and the risk for hypoglycemia increase [10].

Anesthetic or sedative agents may influence glucose homeostasis perioperatively in patients undergoing surgery, by reducing catabolic hormone secretion indirectly, or by changing insulin secretion directly. The latter mechanism is relevant only in those patients with some residual insulin secretion (patients with Type 2 diabetes) [3].

Fragen et al. [37] demonstrated that etomidate inhibits adrenal steroid genesis, and may induce a decrease in the glycemic response to surgery. In general, $\gamma$-aminobutyric acid (GABA) agonists reduce the secretion of adrenocorticotrophic hormone (ACTH) and consequently cortisol, and stimulate basal secretion of growth hormone (GH). Several studies have investigated modifications of the metabolic and hormonal responses to surgery by benzodiazepines, such as midazolam. Desborough et al. [38] examined the effects of midazolam on hormonal and metabolic responses to surgery. In this study, midazolam was infused at a dose of $0.42 \mathrm{mg} / \mathrm{kg}$, followed by an infusion of $0.125 \mathrm{mg} /$ $\mathrm{kg}$ for $1 \mathrm{~h}$. They found that infusion of midazolam resulted in decreased secretion of cortisol and insulin and increased GH secretion [36]. High doses of opiates induce not only hemodynamic, but also hormonal and metabolic instability [39]. Barbiturates (BBT) are depressors of global metabolism without production of lactate accumulation. They have no relevant effects on systemic glucose regulation [40]. Propofol causes a minimal elevation in extracellular glucose [40]. Unlike isoflurane, it stimulates insulin secretion and, therefore, is less likely to generate hyperG [41]. Ketamine produces a mild-to-moderate rise in cerebral oxygen and glucose consumption. It also increases cerebral lactate levels moderately [40].

These anesthetic agents effectively inhibit the entire sympathetic nervous system and hypothalamic-pituitary axis. Inhibition of the catabolic hormone response to surgery may be beneficial, particularly in diabetic patients.

In vitro, volatile anesthetics such as halothane, enflurane and isoflurane inhibit insulin response to glucose in a reversible and dosedependent manner [42-44]. A clinical study by Diltoer and Camu [45] showed that glucose tolerance was impaired by isoflurane. In an experimental study [46], halogenated anesthetic agents, such as halothane or sevoflurane, produced greater negative inotropic effects in diabetic patients compared with non-diabetes, possibly because diabetes exacerbates anesthetic-induced alterations in troponin-tropomyosin complex activity. The effect of propofol on insulin secretion is unclear. Alterations in the pharmacokinetics or pharmacodynamics of propofol in rats with diabetes mellitus have been reported from an experimental study [47], but no data show whether or not propofol can alter lipid clearance from the circulation. At present, it remains uncertain which anesthetic agents facilitate adequate glucose control and hemodynamic stability during the perioperative period [36].

\section{Considerations and Conclusions}

Anesthesiologists should pay attention to not only the efficacy and risk of IIT during the perioperative period in diabetes neurosurgical patients, but also the effects of anesthetic agents on glucose equilibrium [36].

Microdialysis researches in patients undergoing IIT therapy providing significant insight into regional alterations of glucose metabolism in injured brain tissue. Microdialysis has been referred to as multimodal neuroprotective monitoring [4]. However, Microdialysis has its limitations, despite scientific advances in making microdialysis probes smaller and more efficient; the invasive nature of this technique still poses some practical and ethical limitations. For example, it has been shown that implantation of a microdialysis probe can alter tissue morphology, resulting in disturbed microcirculation, rate of metabolism or integrity of physiological barriers, such as the blood-brain barrier. While acute reactions to probe insertion, such as implantation traumas, require sufficient recovery time, additional factors, such as necrosis, inflammatory responses, or wound healing processes have to be taken into consideration for long-term sampling as they may influence the experimental outcome. From a practical perspective, it has been suggested to perform microdialysis experiments within an optimal time window, usually $24-48$ hours after probe insertion $[48,49]$. We should explore a better technology to monitor the change of blood glucose during the perioperative period. New technology may avoid the occurrence of hypoglycemia.

In a study of 918 consecutive neurosurgical patients at the University of Michigan Hospitals, Davis et al. found that increased preoperative blood glucose was associated with higher risk for postoperative complications, longer hospital stays, and longer neurosurgical ICU stays. Moreover, increasing preoperative blood glucose predicted both complication risk and ICU and hospital stay in a dose-response fashion, such that incrementally higher blood glucose predicted incrementally higher complication risk and longer ICU and hospital stay [50].

It can be seen from the Figure 1 that preoperative blood glucose above $120 \mathrm{mg} / \mathrm{dl}$ predicted risk for postoperative complications,

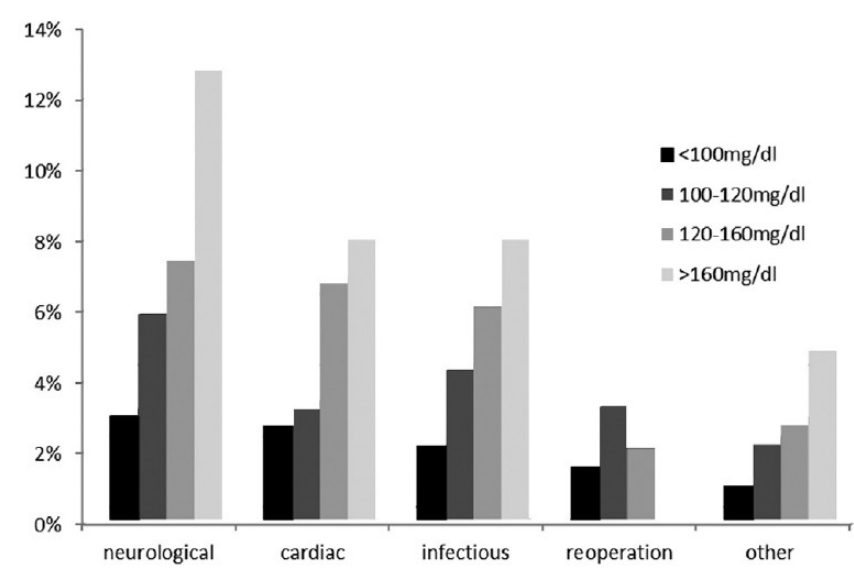

Figure 1: 30-day postoperative complication rates as a function of preoperative blood glycemia values among 918 neurosurgical patients at the University of Michigan Hospitals [50]. 
Citation: Tao J, Youtan L (2013) Impact of Anesthesia on Systemic and Cerebral Glucose Metabolism in Diabetes Patients Undergoing Neurosurgery "Updates of Diabetes and Neurosurgical Anesthesia". J Diabetes Metab 4: 297. doi:10.4172/2155-6156.1000297

increased length of hospital stay, and increased length of neourosurgical ICU stay in a dose-response fashion following neurosurgical intervention. Tight glycemic control peri-operatively may lead to reduced risk for postoperative complications and more rapid recovery following neurosurgery. Randomized controlled trials of the effects of tight glycemic control on post-neurosurgical outcomes among hyperglycemics are in order [45].

Glucose is the only resource for brain energy metabolism. IIT is a double-edged swords, it should be carefully considered in diabetes neurosurgical patients. Extreme hyperglycemia and hypoglycemia episodes must be avoided (4.4 7.0/5.0 8.7 mmol are suggested) [51]. Frequently and routinely monitor of glucose level are necessary for diabetes patients undergoing neurosurgery

Surgical stimulation could affect patient stress response and metabolic/endocrine system and subsequently lead to increase of blood glucose level. Some anesthetics as well as some therapeutic agents have effects on systemic and cerebral glucose/oxygen consumption, and thus may affect blood glucose level. Adequate depth of anesthesia could prevent surgical induced stress response and a balanced anesthesia should be more recommended in diabetes patients.

Anesthesiologists play a very important role during the perioperative period in diabetes neurosurgical patients. Systemic and cerebral metabolism of glucose in diabetes neurosurgical patients should be measured during the operation, and a better follow-up is very necessary. Good blood glucose control protocols should be developed according to the patient's prognosis.

\section{References}

1. (2008) The global challenge of diabetes. Lancet $371: 1723$.

2. Nicholson G, Hall GM (2011) Diabetes mellitus: new drugs for a new epidemic. $\mathrm{Br}$ J Anaesth 107: 65-73.

3. Frisch A, Chandra P, Smiley D, Peng L, Rizzo M, et al. (2010) Prevalence and clinical outcome of hyperglycemia in the perioperative period in noncardiac surgery. Diabetes Care 33: 1783-1788.

4. Atkins JH, Smith DS (2009) A review of perioperative glucose control in the neurosurgical population. J Diabetes Sci Technol 3: 1352-1364.

5. Ooi YC, Dagi TF, Maltenfort M, Rincon F, Vibbert M, et al. (2012) Tight glycemic control reduces infection and improves neurological outcome in critically ill neurosurgical and neurological patients. Neurosurgery 71: 692-702.

6. Passero S, Ciacci G, Ulivelli M (2003) The influence of diabetes and hyperglycemia on clinical course after intracerebral hemorrhage. Neurology 61: $1351-1356$

7. Godoy DA, Di Napoli M, Rabinstein AA (2010) Treating hyperglycemia in neurocritical patients: benefits and perils. Neurocrit Care 13: 425-438.

8. Lipshutz AK, Gropper MA (2009) Perioperative glycemic control: an evidencebased review. Anesthesiology 110: 408-421.

9. Bilotta F, Giovannini F, Caramia R, Rosa G (2009) Glycemia management in neurocritical care patients: a review. J Neurosurg Anesthesiol 21: 2-9.

10. Kang $H(2012)$ Current therapeutic agents and anesthetic considerations for diabetes mellitus. Korean J Anesthesiol 63: 195-202.

11. Robertshaw HJ, Hall GM (2006) Diabetes mellitus: anaesthetic management Anaesthesia 61: 1187-1190.

12. Godoy DA, Di Napoli M, Biestro A, Lenhardt R (2012) Perioperative glucose control in neurosurgical patients. Anesthesiol Res Pract 2012: 690362.

13. Wang YY, Lin SY, Chuang YH, Chen CJ, Tung KC, et al. (2011) "Adipose proinflammatory cytokine expression through sympathetic system is associated with hyperglycemia and insulin resistance in a rat ischemic stroke model. Am J Physiol 300: 155-163.

14. Wilcockson DC, Campbell SJ, Anthony DC, Perry VH (2002) The systemic and local acute phase response following acute brain injury. J Cereb Blood Flow Metab 22: 318-326.
15. Harada S, Fujita-Hamabe W, Tokuyama S (2011) Effect of orexin-A on postischemic glucose intolerance and neuronal damage. J Pharmacol Sci 115: 155163.

16. Allport LE, Butcher KS, Baird TA, MacGregor L, Desmond PM, et al. (2004) Insular cortical ischemia is independently associated with acute stress hyperglycemia. Stroke 35: 1886-1891.

17. Klein JP, Waxman SG (2003) The brain in diabetes: molecular changes in neurons and their implications for end-organ damage. Lancet Neurol 2: 548554

18. Moghissi ES, Korytkowski MT, DiNardo M, Einhorn D, Hellman R, et al. (2009) American Association of Clinical Endocrinologists and American Diabetes Association consensus statement on inpatient glycemic control. Diabetes Care 32: $1119-1131$

19. Bilotta F, Rosa G (2010) Glucose management in the neurosurgical patient: are we yet any closer? Curr Opin Anaesthesiol 23: 539-543.

20. Graffagnino C, Gurram AR, Kolls B, Olson DM (2010) Intensive insulin therapy in the neurocritical care setting is associated with poor clinical outcomes. Neurocrit Care 13: 307-312.

21. Green DM, O'Phelan KH, Bassin SL, Chang CW, Stern TS, et al. (2010) Intensive versus conventional insulin therapy in critically ill neurologic patients. Neurocrit Care 13: 299-306.

22. Lupi A, Bertagnoni G, Salgarello M, Orsolon P, Malfatti V, et al. (2007) Cerebella vermis relative hypermetabolism: an almost constant $P E T$ finding in an injured brain. Clin Nucl Med 32: 445-451.

23. van den Berghe $G$, Wouters $P$, Weekers $F$, Verwaest $C$, Bruyninckx F, et al (2001) Intensive insulin therapy in critically ill patients. N Engl J Med 345: 13591367.

24. Van den Berghe G, Schoonheydt K, Becx P, Bruyninckx F, Wouters PJ (2005) Insulin therapy protects the central and peripheral nervous system of intensive care patients. Neurology 64: 1348-1353.

25. Vespa PM (2008) Intensive glycemic control in traumatic brain injury: what is the ideal glucose range? Crit Care 12: 175.

26. Van den Berghe G, Wilmer A, Hermans G, Meersseman W, Wouters PJ, et al (2006) Intensive insulin therapy in the medical ICU. N Engl J Med 354: 449-461.

27. Vespa P, Boonyaputthikul R, McArthur DL, Miller C, Etchepare M, et al. (2006) Intensive insulin therapy reduces microdialysis glucose values without altering glucose utilization or improving the lactate/pyruvate ratio after traumatic brain injury. Crit Care Med 34: 850-856.

28. Schlenk F, Graetz D, Nagel A, Schmidt M, Sarrafzadeh AS (2008) Insulinrelated decrease in cerebral glucose despite normoglycemia in aneurysmal subarachnoid hemorrhage. Crit Care 12: R9.

29. Zygun DA, Steiner LA, Johnston AJ, Hutchinson PJ, Al-Rawi PG, et al. (2004) Hyperglycemia and brain tissue $\mathrm{pH}$ after traumatic brain injury. Neurosurgery 55: $877-881$

30. Bilotta F, Spinelli A, Giovannini F, Doronzio A, Delfini R, et al. (2007) The effect of intensive insulin therapy on infection rate, vasospasm, neurologic outcome and mortality in neurointensive care unit after intracranial aneurysm clipping in patients with acute subarachnoid hemorrhage: a randomized prospective pilot trial. J Neurosurg Anesthesiol 19: 156-160.

31. Meier R, Béchir M, Ludwig S, Sommerfeld J, Keel M, et al. (2008) Differentia temporal profile of lowered blood glucose levels ( 3.5 to $6.5 \mathrm{mmol} / /$ versus 5 to 8 $\mathrm{mmol} / \mathrm{l})$ in patients with severe traumatic brain injury. Crit Care 12: R98.

32. Girard M, Schricker T (2011) Perioperative glucose control: living in uncertain times--continuing professional development. Can J Anaesth 58: 320-329.

33. Atkins JH, Smith DS (2009) A review of perioperative glucose control in the neurosurgical population. J Diabetes Sci Technol 3: 1352-1364

34. Schuster JM, Rechtine G, Norvell DC, Dettori JR (2010) The influence of perioperative risk factors and therapeutic interventions on infection rates after spine surgery: a systematic review. Spine (Phila Pa 1976) 35: S125-137.

35. (1998) Intensive blood-glucose control with sulphonylureas or insulin compared with conventional treatment and risk of complications in patients with type 2 diabetes (UKPDS 33). UK Prospective Diabetes Study (UKPDS) Group. Lancet 352: 837-853.

36. (1998) Tight blood pressure control and risk of macrovascular and microvascular complications in type 2 diabetes: UKPDS 38. UK Prospective Diabetes Study Group. BMJ 317: 703-713. 
Citation: Tao J, Youtan L (2013) Impact of Anesthesia on Systemic and Cerebral Glucose Metabolism in Diabetes Patients Undergoing Neurosurgery "Updates of Diabetes and Neurosurgical Anesthesia". J Diabetes Metab 4: 297. doi:10.4172/2155-6156.1000297

Page 5 of 5

37. Fragen RJ, Shanks CA, Molteni A, Avram MJ (1984) Effects of etomidate on hormonal responses to surgical stress. Anesthesiology 61: 652-656.

38. Desborough JP, Hall GM, Hart GR, Burrin JM (1991) Midazolam modifies pancreatic and anterior pituitary hormone secretion during upper abdominal surgery. Br J Anaesth 67: 390-396.

39. Finney SJ, Zekveld C, Elia A, Evans TW (2003) Glucose control and mortality in critically ill patients. JAMA 290: 2041-2047.

40. Kadoi $Y$ (2012) Blood glucose control in the perioperative period. Minerva Anestesiol 78: 574-595

41. Hall GM, Lacoumenta S, Hart GR, Burrin JM (1990) Site of action of fentany in inhibiting the pituitary-adrenal response to surgery in man. $\mathrm{Br} \mathrm{J}$ Anaesth 65 : 251-253

42. Horn T, Klein J (2010) Lactate levels in the brain are elevated upon exposure to volatile anesthetics: a microdialysis study. Neurochem Int 57: 940-947.

43. Tanaka K, Kawano T, Tsutsumi YM, Kinoshita M, Kakuta N, et al. (2011) Differential effects of propofol and isoflurane on glucose utilization and insulin secretion. Life Sci 88: 96-103.

44. Desborough JP, Jones PM, Persaud SJ, Landon MJ, Howell SL (1993) Isoflurane inhibits insulin secretion from isolated rat pancreatic islets of Langerhans. Br J Anaesth 71: 873-876.
45. Diltoer M, Camu F (1988) Glucose homeostasis and insulin secretion during isoflurane anesthesia in humans. Anesthesiology 68: 880-886.

46. David JS, Tavernier B, Amour J, Vivien B, Coriat P, et al. (2004) Myocardia effects of halothane and sevoflurane in diabetic rats. Anesthesiology 100: 11791187.

47. Leal N, Calvo R, Agrad FZ, Lukas JC, de la Fuente L, et al. (2005) Altered doseto-effect of propofol due to pharmacokinetics in rats with experimental diabetes mellitus. J Pharm Pharmacol 57: 317-325.

48. Di Chiara G, Tanda G, Carboni E (1996) Estimation of in-vivo neurotransmitte release by brain microdialysis: the issue of validity. Behav Pharmacol 7: 640657

49. Westerink BH, Damsma G, Rollema H, De Vries JB, Horn AS (1987) Scope and limitations of in vivo brain dialysis: a comparison of its application to various neurotransmitter systems. Life Sci 41: 1763-1776.

50. Davis MC, Ziewacz JE, Sullivan SE, El-Sayed AM (2012) Preoperative hyperglycemia and complication risk following neurosurgical intervention: $A$ study of 918 consecutive cases. Surg Neurol Int 3: 49.

51. Ruquan GMH (2011) Progress of Perioperative Glucose Control in the Neurosurgical patients. Chin J Clinicians 5. 